\title{
Electrochemical Controlled Synthesis of Au/Ni Nano-arrays and its Sintering with Ag NPs Paste
}

\author{
Zhen Zheng ${ }^{1}$, Fan Yang ${ }^{1}$, Wei Liu ${ }^{1, *}$, Fanyu Meng ${ }^{2}$, Rong An ${ }^{1,3}$, Wei Zhang ${ }^{l}$, Haibo Wang ${ }^{1}$, \\ Yiping Wang ${ }^{1}$, Chunqing Wang ${ }^{1,3, *}$ \\ ${ }^{1}$ State Key Laboratory of Advanced Welding and Joining, Harbin Institute of Technology, Harbin \\ 150001, China \\ ${ }^{2}$ Department of Environmental Hygiene, School of Public Health, Harbin Medical University, Harbin \\ 150081, China \\ ${ }^{3}$ Key Laboratory of Micro-systems and Micro-structures Manufacturing, Ministry of Education, \\ Harbin Institute of Technology, Harbin 150080, China \\ *E-mail: w__liu@hit.edu.cn, wangcq@hit.edu.cn
}

doi: $10.20964 / 2020.08 .80$

Received: 8 March 2019 / Accepted: 18 June 2020 / Published: 10 July 2020

\begin{abstract}
The microstructure gap between the nanopaste and the conventional pad led to the high practical connection temperature. In this paper, we focused on the preparation of the different microstrucutre $\mathrm{Ni}$ nanocone arrays and their sintering behaviors. The electrochemical behavior of ethylenediamine hydrochloride $(\mathrm{EDH})$ on the Ni deposition was studied. The SEM, XRD, linear polarization and impedance spectroscopy analysis results showed that EDH could facilitate the oriented growth of nickel and decrease the polarization of electrodeposition. Different Ni nanocone arrays were prepared by tuning the EDH concentration, current density and solution temperature. The pads coated with $\mathrm{Ni} / \mathrm{Au}$ nanocone arrays were connected with Ag NPs paste. The shear test result showed that the surface array microstructure played a significant role in enhancing the joint strength. When the nanocone bottom diameter was $150 \mathrm{~nm}$, the joint hold the best shear strength.
\end{abstract}

Keywords: Ni nanocone arrays, Ethylenediamine hydrochloride, Ag NPs, Sinter, Shear strength

\section{FULL TEXT}

(C) 2020 The Authors. Published by ESG (www.electrochemsci.org). This article is an open access article distributed under the terms and conditions of the Creative Commons Attribution license (http://creativecommons.org/licenses/by/4.0/). 